\title{
A00-39808
}

AIAA-2000-4424

\section{MARS AEROCAPTURE USING BANK MODULATION}

\author{
Nguyen X. Vinh* \\ Department of Aerospace Engineering \\ The University of Michigan \\ Ann Arbor, Michigan 48109-2140 \\ and \\ Wyatt R. Johnson ${ }^{\dagger}$ and James M. Longuski ${ }^{\ddagger}$ \\ School of Aeronautics and Astronautics, Purdue University \\ West Lafayette, Indiana 47907-1282
}

\begin{abstract}
In this paper we investigate using bank modulation of a lifting body to capture a spacecraft into orbit in a single atmospheric flythrough. Aerocapture without lift is difficult to achieve because ballistic flythroughs are highly sensitive to the atmospheric entry angle. Lifting bodies are more robust and provide a simple means of control through bank modulation. First and second order analytic solutions to the equations of motion are derived, and are compared against precise numerical integration. Control laws based on the analytic theory guide the spacecraft to the desired exit conditions.
\end{abstract}

\section{Nomenclature}

$C_{D}=$ Coefficient of drag

$C_{L}=$ Coefficient of lift

$E=$ Lift and bank control parameter

$e=$ Eccentricity

$g=$ Gravitational acceleration, $\mathrm{m} / \mathrm{s}^{2}$

$m=$ Mass of spacecraft, $\mathrm{kg}$

$R=$ Radius of atmospheric entry, $\mathrm{km}$

$r=$ Radius of spacecraft's orbit, $\mathrm{km}$

$S=$ Planform area of spacecraft's wing, $m^{2}$

$s=$ Nondimensional arc length of spacecraft's trajectory

$V=$ Velocity of spacecraft relative to planet, $\mathrm{km} / \mathrm{s}$

$x=$ Nondimensional speed parameter

$y=$ Nondimensional altitude parameter

$\alpha=$ Apoapsis in Mars radii

$\beta=$ Inverse scale height of atmosphere, $k m^{-1}$

$\delta=$ Nondimensional entry speed parameter

$\gamma=$ Flight path angle, rad

$\epsilon=$ Nondimensional entry altitude parameter

$\rho=$ Atmospheric density, $\mathrm{kg} / \mathrm{m}^{3}$

$\sigma=$ Bank angle, rad

*Professor Emeritus.

†Doctoral Candidate, Member AIAA.

$\ddagger$ Professor, Associate Fellow AIAA, Member AAS.

Copyright (C) 2000 by Nguyen X. Vinh, Wyatt R. Johnson, and James M. Longuski. Published by the American Institute of Aeronautics and Astronautics, Inc. with permission.

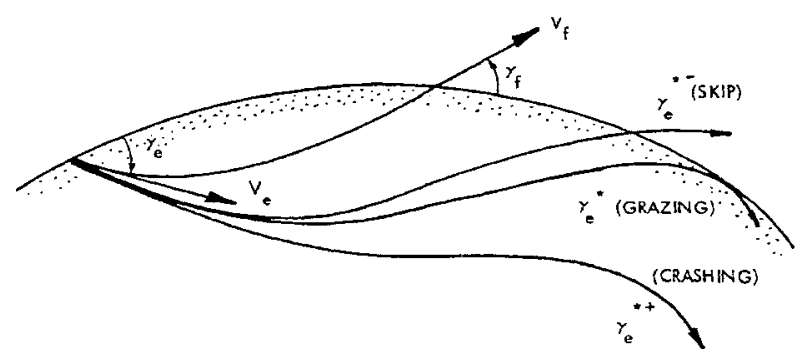

Fig. 1 Geometry of ballistic skip trajectory with behavior near critical entry angle, $\gamma_{e}^{* 1}$

\section{Subscripts}

$e=$ atmospheric entry condition

$f=$ atmospheric exit condition

$b=$ at bottom of atmospheric trajectory

\section{Introduction}

$\mathrm{D}$ URING aerocapture, a spacecraft flies through the atmosphere of a planet to achieve orbit in a single pass. Unfortunately, capture for ballistic (nonlifting) vehicles is extremely sensitive to the atmospheric entry angle ${ }^{1,2}$ - too steep or too shallow and the spacecraft will crash or skip out, as shown in Fig. 1. Several missions (such as Magellan and the Mars Global Surveyor) have instead employed aerobraking where multiple passes over an extended period of time gradually adjust the orbit; but in these cases an impulsive maneuver is first required to capture the spacecraft. The Mars 2003/2005 Sample Return Mission ${ }^{3}$ is considering using a lifting body to satisfy operational constraints by capturing into 
the final orbit in one passage. The advantage of the lifting body is that it is less sensitive to variation in the entry angle and the drag is easily modulated.

In this paper we consider bank modulation ${ }^{4}$ to control the drag during the atmospheric passage to achieve the exit condition for ascent into the final orbit. We derive a second order analytic solution for the flythrough trajectory and develop two control laws to modulate the bank so the spacecraft will exit the atmosphere with the desired apoapsis.

\section{Equations of Motion}

For three-dimensional gliding flight, with lift and bank, the equations of motion are: ${ }^{5}$

$$
\begin{aligned}
\dot{r} & =V \sin \gamma \\
\dot{\theta} & =\frac{V \cos \gamma \cos \psi}{r \cos \phi} \\
\dot{\phi} & =\frac{V \cos \gamma \sin \psi}{r} \\
\dot{V} & =\frac{-D}{m}-g \sin \gamma \\
V \dot{\gamma} & =\frac{L \cos \sigma}{m}-g \cos \gamma+\frac{V^{2} \cos \gamma}{r} \\
V \dot{\psi} & =\frac{L \sin \sigma}{m \cos \gamma}-\frac{V^{2} \cos \gamma \cos \psi \tan \phi}{r}
\end{aligned}
$$

In this research, we are only concerned with parameters directly related to achieving orbit (and not the particular final three-dimensional orbit the spacecraft will achieve). Thus, the two state variables of the most interest to us are $r$ and $V$. Examining Eqs. 1 and 4, we see that analyzing another state variable, $\gamma$ is also required. Thus, the relevant equations of motion are:

$$
\begin{aligned}
\dot{r} & =V \sin \gamma \\
\dot{V} & =-\frac{\rho S V^{2} C_{D}}{2 m}-g \sin \gamma \\
V \dot{\gamma} & =\frac{\rho S V^{2} C_{L}}{2 m} \cos \sigma-g \cos \gamma+\frac{V^{2}}{r} \cos \gamma
\end{aligned}
$$

To ease the analysis, we nondimensionalize the equations of motion, Eqs. 7-9. We use the dimensionless arc length $s$ to replace time $t$ :

$$
d s=\sqrt{\frac{\beta}{R}} V d t
$$

We nondimensionalize the altitude with:

$$
y=\frac{\rho}{\rho_{e}}=\exp (-\beta h)
$$

The quantity $h$ in Eq. 11 is the altitude of the spacecraft relative to the edge of the atmosphere. Thus, $h \leq 0$. Finally, we nondimensionalize the speed by:

$$
x=\ln \left(\frac{V_{e}}{V}\right)^{2}
$$

Equations 11 and 12 define new dimensionless variables in terms of the dimensional entry parameters, $\rho_{e}$ and $V_{e}$. These two are defined in terms of two more dimensionless quantities:

$$
\begin{aligned}
\epsilon & =\sqrt{\frac{R}{\beta}} \frac{\rho_{e} S C_{D}}{m} \\
\delta & =\frac{\mu / R}{V_{e}^{2}}
\end{aligned}
$$

With these definitions, we can now nondimensionalize Eqs. 7-9, yielding:

$$
\begin{aligned}
\frac{d y}{d s}= & -\sqrt{\beta R} y \sin \gamma \\
\frac{d x}{d s}= & \epsilon y+\frac{2 \delta e^{x}}{\sqrt{\beta R}}\left(\frac{R}{r}\right)^{2} \sin \gamma \\
\frac{d \gamma}{d s}= & \frac{\epsilon}{2} y\left(\frac{C_{L}}{C_{D}}\right) \cos \sigma+\frac{\cos \gamma}{\sqrt{\beta R}}\left(\frac{R}{r}\right) \\
& \times\left[1-\delta e^{x}\left(\frac{R}{r}\right)\right]
\end{aligned}
$$

The term $R / r$ can be expanded into the geometric series:

$$
\begin{aligned}
\frac{R}{r} & =\frac{1}{1+\frac{\Delta r}{R}} \\
& =1-\frac{\Delta r}{R}+\left(\frac{\Delta r}{R}\right)^{2}-\cdots
\end{aligned}
$$

By neglecting $\Delta r / R$, the resulting error is on the same order as neglecting the Coriolis force - the assumption made for a non-rotating planet. Hence, we shall consider as exact equations of motion the set Eqs. 19-21:

$$
\begin{aligned}
& \frac{d y}{d s}=-\sqrt{\beta R} y \sin \gamma \\
& \frac{d x}{d s}=\epsilon y+\frac{2 \delta e^{x}}{\sqrt{\beta R}} \sin \gamma \\
& \frac{d \gamma}{d s}=\frac{\epsilon}{2} y\left(\frac{C_{L}}{C_{D}}\right) \cos \sigma+\frac{\cos \gamma}{\sqrt{\beta R}}\left(1-\delta e^{x}\right)
\end{aligned}
$$




\section{First-order solution}

We can omit the component of the gravity acceleration along the tangent to the flight path. Furthermore, by Eggers' assumption ${ }^{5,6}$ the combined gravitational force and centrifugal force along the normal to the flight path are assumed to be small and balanced out as compared to the lift force. Then, we have the reduced set of equations:

$$
\begin{aligned}
& \frac{d y}{d s}=-\sqrt{\beta R} y \sin \gamma \\
& \frac{d x}{d s}=\epsilon y \\
& \frac{d \gamma}{d s}=\frac{\epsilon}{2} y E
\end{aligned}
$$

where, for convenience of notion, we have taken

$$
E=\left(\frac{C_{L}}{C_{D}}\right) \cos \sigma
$$

as the lift-and-bank control parameter. In the analytic solution, we assume a constant lift-to-drag ratio, but for the control law, we can modulate the bank angle by varying $E$. The reduced equations allow for the first-order solution:

$$
\sqrt{\beta R}\left(\cos \gamma-\cos \gamma_{e}\right)=\frac{\epsilon E}{2}(y-1)
$$

Equation 26 shows the variation of the "altitude variable" (density ratio) as a function of the flight path angle. At exit, we have $y_{f}=1$, and thus:

$$
\cos \gamma_{f}=\cos \gamma_{e}
$$

where the entry angle is negative, while the exit angle is positive, but equal in magnitude. In reality, the exit angle is slightly less as will be shown in the second order solution.

For the speed variation, we combine Eqs. 23 and 24 to have:

$$
\frac{d x}{d \gamma}=\frac{2}{E}
$$

Hence

$$
x=\frac{2\left(\gamma-\gamma_{e}\right)}{E}
$$

or

$$
\frac{V}{V_{e}}=\exp \left[\frac{\gamma_{e}-\gamma}{E}\right]
$$

Since at exit, $\gamma_{f}=-\gamma_{e}$, then

$$
V_{f}=V_{e} \exp \left[\frac{2 \gamma_{e}}{E}\right]
$$

The first-order solutions allow the computation of the elements at the lowest point of the trajectory where $\gamma=0$. From Eqs. 26 and 30, we have, at the bottom:

$$
y_{b}=1+\frac{4 \sqrt{\beta R}}{\epsilon E} \sin ^{2} \frac{\gamma_{e}}{2}
$$

and

$$
V_{b}=V_{e} \exp \left[\frac{\gamma_{e}}{E}\right]
$$

Therefore, we have the geometric mean:

$$
V_{b}^{2}=V_{e} V_{f}
$$

Notice that, from Eq. 11 and the exponential atmosphere assumption, the variation in altitude from the entry point is:

$$
\frac{h}{R}=-\frac{1}{\beta R} \ln y
$$

\section{Second-order solution}

Now we derive a set of second-order solutions, where we obtain a more precise solution by including more terms from the equations of motion (EOMs) than the first-order solution. We first use $y$ as the independent variable to replace the arc length $s$. By using Eq. 19 in 21, we have the equation for the flight path angle:

$$
-\sqrt{\beta R} \sin \gamma \frac{d \gamma}{d y}=\frac{\epsilon}{2} E+\frac{\cos \gamma}{y \sqrt{\beta R}}\left(1-\delta e^{x}\right)
$$

On the right hand side of Eq. 36, we approximate $\cos \gamma \approx 1$ and expand the exponential to the first order by $e^{x} \approx 1+x$ to yield:

$$
\frac{d}{d y}(\sqrt{\beta R} \cos \gamma)=\frac{\epsilon}{2} E+\frac{1-\delta}{y \sqrt{\beta R}}-\frac{\delta x}{y \sqrt{\beta R}}
$$

Upon integrating from $\gamma=\gamma_{e}$ and $y=1$, we have the solution for the flight path angle:

$$
\begin{aligned}
\sqrt{\beta R}\left(\cos \gamma-\cos \gamma_{e}\right)= & \frac{\epsilon E}{2}(y-1)+\frac{1-\delta}{\sqrt{\beta R}} \ln y \\
& -\frac{\delta}{\sqrt{\beta R}} I(y)
\end{aligned}
$$

where the integral in the last term is:

$$
I(y)=\int_{1}^{y} \frac{x(y) d y}{y}
$$

As an improved first-order solution, we can neglect the contribution of this integral and consider the change in the flight path angle as a function of the altitude in the form:

$$
\sqrt{\beta R}\left(\cos \gamma-\cos \gamma_{e}\right)=\frac{\epsilon E}{2}(y-1)+\frac{1-\delta}{\sqrt{\beta R}} \ln y
$$


For the speed variable $x$, we substitute Eq. 19 into Eq. 20 to yield:

$$
\frac{d x}{d s}=\epsilon y-\frac{2 \delta e^{x}}{\beta R y} \frac{d y}{d s}
$$

Expanding $e^{x}$ to the first order gives:

$$
\frac{d x}{d s}=\epsilon y-\frac{2 \delta}{\beta R y} \frac{d y}{d s}-\frac{2 \delta}{\beta R}\left(\frac{x}{y}\right) \frac{d y}{d s}
$$

Next, we obtain $\epsilon y$ from Eq. 21 using $\cos \gamma \approx 1$ and $e^{x} \approx 1$ as:

$$
\epsilon y=\frac{2}{E}\left[\frac{d \gamma}{d s}-\frac{1}{\sqrt{\beta R}}(1-\delta)\right]
$$

Substituting Eq. 43 into 42 yields:

$$
\begin{aligned}
\frac{d x}{d s}= & \frac{2}{E} \frac{d \gamma}{d s}-\frac{2}{\sqrt{\beta R E}}(1-\delta) \\
& -\frac{2 \delta}{\beta R y} \frac{d y}{d s}-\frac{2 \delta}{\beta R}\left(\frac{x}{y}\right) \frac{d y}{d s}
\end{aligned}
$$

Integrating Eq. 44 yields:

$$
\begin{aligned}
x= & \frac{2\left(\gamma-\gamma_{e}\right)}{E}-\frac{2 \delta}{\beta R} \ln y-\frac{2(1-\delta)}{\sqrt{\beta R} E} s \\
& -\frac{2 \delta}{\beta R} I(y)
\end{aligned}
$$

It remains to evaluate the arc length $s$, and the integral $I(y)$. Since these terms contribute to the approximate solution, we shall use the first-order solutions for $x$ and $y$ for their evaluation.

In the first-order derivation we approximate $d \gamma / d s$ by only one term (Eq. 21), whereas the original EOM had three terms (Eq 24). In the secondorder approximation, we make the approximation $\delta e^{x} \approx \delta+\delta x \approx \delta$. This approximation is exact at initial conditions, and loses its accuracy as $x$ increases. Equation 21 is thus approximated by:

$$
\frac{d \gamma}{d s}=\frac{\epsilon E y}{2}+\frac{1-\delta}{\sqrt{\beta R}}
$$

We use the first order solution, Eq. 40, to evaluate $E \epsilon y / 2$ in Eq. 46. Also, we approximate $\ln y \approx k(y-$ 1) for some value $\mathrm{k}$. Making these substitutions, we have:

$$
\sqrt{\beta R}\left(\cos \gamma-\cos \gamma_{e}\right)=\left[\frac{\epsilon E}{2}+\frac{k(1-\delta)}{\sqrt{\beta \bar{R}}}\right](y-1)
$$

The approximation $\ln y \approx k(y-1)$ is exact for two values of $y$ - one of them is $y=1$ while the other may be chosen by appropriate choice of $k$. We let the other value correspond to the spacecraft's lowest point in the trajectory - when $\gamma=0$, and $y=y_{\max } \equiv y_{b}$. Thus,

$$
k=\frac{\ln y_{b}}{y_{b}-1}
$$

The value of $y_{b}$ can be solved numerically from Eq. 40 , by allowing $\gamma=0$. We note that Eq. 47 can be rewritten in a simpler form by defining a new constant:

$$
k_{1}=\frac{\epsilon E}{2 \sqrt{\beta R}}+\frac{k(1-\delta)}{\beta R}
$$

Equation 47 is solved for $y$ and written as:

$$
y=\frac{1}{k_{1}}\left(\cos \gamma-\cos \gamma_{e}+k_{1}\right)
$$

Note that Eq. 50 only provides a rough expression for $y$ that is used only in helping derive an expression for s. Eq. 40 provides the second-order approximation for $y$. Substituting Eq. 50 into Eq. 46, we have:

$$
\frac{d \gamma}{d s}=\frac{\epsilon E}{2 k_{1}}\left(\cos \gamma-\cos \gamma_{e}+k_{1}\right)+\frac{1-\delta}{\sqrt{\beta R}}
$$

Equation 51 can be rewritten in the form:

$$
d s=\frac{A d \gamma}{\cos \gamma-\cos \gamma_{0}}
$$

where the constants $\mathrm{A}$ and $\gamma_{0}$ are defined by:

$$
\begin{aligned}
A= & \frac{1}{\sqrt{\beta R}}\left[1+\frac{2 k(1-\delta)}{\epsilon E \sqrt{\beta R}}\right] \\
\cos \gamma_{0}= & \cos \gamma_{e}-\left[\frac{\epsilon E}{2 \sqrt{\beta R}}+\frac{(1+k)(1-\delta)}{\beta R}\right. \\
& \left.+\frac{2 k(1-\delta)^{2}}{\epsilon E(\beta R)^{3 / 2}}\right]
\end{aligned}
$$

We choose $\gamma_{0}$ to be positive, so that $\gamma_{0}>\left|\gamma_{e}\right|$. Equation 52 can be integrated to yield the second order solution for $s$ :

$$
s=\frac{A}{\sin \gamma_{0}} \ln \left[\frac{\left(\tan \frac{\gamma_{0}}{2}+\tan \frac{\gamma}{2}\right)\left(\tan \frac{\gamma_{0}}{2}-\tan \frac{\gamma_{e}}{2}\right)}{\left(\tan \frac{\gamma_{0}}{2}-\tan \frac{\gamma}{2}\right)\left(\tan \frac{\gamma_{0}}{2}+\tan \frac{\gamma_{e}}{2}\right)}\right]
$$

This equation will be used to help evaluate the expressions for $I(y)$ and $x$.

To evaluate the $I(y)$ integral, we start with the definition, and then integrate by parts:

$$
\begin{aligned}
I(y) & =\int x \frac{d y}{y} \\
& =\int x d(\ln y) \\
& =x \ln y-\int \ln y d x
\end{aligned}
$$


We approximate $x$ by using the first three terms of Eq. 45. The differential $d x$ is evaluated using Eq. 41. Making these substitutions yields:

$$
\begin{aligned}
I(y)= & \frac{2\left(\gamma-\gamma_{e}\right)}{E} \ln y-\frac{\delta}{\beta R}(\ln y)^{2}-\frac{2(1-\delta)}{\sqrt{\beta R} E} s \ln y \\
& -\frac{2}{E} \int \ln y d \gamma+\frac{2(1-\delta)}{\sqrt{\beta \bar{R}} E} \int \ln y d s
\end{aligned}
$$

We use Eq. 40 to evaluate the first of the two remaining integrals as:

$$
\begin{aligned}
-\frac{2}{E} \int \ln y d \gamma= & \\
& -\frac{2 \sqrt{\beta R}}{E(1-\delta)} \int\left[\sqrt{\beta R}\left(\cos \gamma-\cos \gamma_{e}\right)\right. \\
& \left.-\frac{\epsilon E y}{2}+\frac{\epsilon E}{2}\right] d \gamma \\
= & -\frac{2 \sqrt{\beta R}}{(1-\delta) E}\left[\frac{\epsilon E}{2}-\sqrt{\beta R} \cos \gamma_{e}\right]\left(\gamma-\gamma_{e}\right) \\
& -\frac{2 \beta R}{(1-\delta) E} \int \cos \gamma d \gamma+\frac{\sqrt{\beta R}}{1-\delta} \int \epsilon y d \gamma(58)
\end{aligned}
$$

We make the small angle approximation for $\cos \gamma \approx$ 1 to yield:

$$
\begin{aligned}
& -\frac{2}{E} \int \ln y d \gamma= \\
& \quad-\frac{2 \beta R}{(1-\delta) E}\left[1+\frac{\epsilon E}{2 \sqrt{\beta R}}-\cos \gamma_{e}\right]\left(\gamma-\gamma_{e}\right) \\
& \quad+\frac{\sqrt{\beta R}}{1-\delta} \int \epsilon y d \gamma
\end{aligned}
$$

The integral in Eq. 59 is evaluated using Eq. 50 for $y$. Simplification yields:

$$
\begin{aligned}
& -\frac{2}{E} \int \ln y d \gamma= \\
& \quad-\frac{4 k \beta R\left(1-\cos \gamma_{e}\right)}{2 k(1-\delta)+\epsilon E \sqrt{\beta R}} \frac{\gamma-\gamma_{e}}{E}
\end{aligned}
$$

We evaluate the second integral from Eq. 57 by using $\ln y$ from Eq. 40:

$$
\begin{aligned}
& \frac{2(1-\delta)}{E \sqrt{\beta R}} \int \ln y d s= \\
& \quad \frac{2}{E} \int\left[\sqrt{\beta R}\left(\cos \gamma-\cos \gamma_{e}\right)-\frac{\epsilon E}{2} y+\frac{\epsilon E}{2}\right] d s \\
& =\frac{2 \sqrt{\beta R}}{E}\left(\frac{\epsilon E}{2 \sqrt{\beta R}}-\cos \gamma_{e}\right) s \\
& \quad+\frac{2 \sqrt{\beta R}}{E} \int \cos \gamma d s-\int \epsilon y d s \\
& =\frac{2 \sqrt{\beta R}}{E}\left(\frac{\epsilon E}{2 \sqrt{\beta R}}-\cos \gamma_{e}\right) s
\end{aligned}
$$

$$
\begin{aligned}
& +\frac{2 \sqrt{\beta R}}{E} \int\left(\cos \gamma-\cos \gamma_{0}\right) d s \\
& +\frac{2 \sqrt{\beta R}}{E} \cos \gamma_{0} s-\int \epsilon y d s
\end{aligned}
$$

To complete the integration, we use Eq. 52 for $\left(\cos \gamma-\cos \gamma_{0}\right) d s$ and Eq. 43 for $\epsilon y$, we have:

$$
\begin{aligned}
& \frac{2(1-\delta)}{E \sqrt{\beta R}} \int \ln y d s= \\
& \quad \frac{2 \sqrt{\beta R}}{E}\left(\frac{\epsilon E}{2 \sqrt{\beta R}}-\cos \gamma_{e}\right) s+\frac{2 A \sqrt{\beta R}}{E}\left(\gamma-\gamma_{e}\right) \\
& \quad+\frac{2 \sqrt{\beta R}}{E} \cos \gamma_{0} s-\frac{2}{E}\left(\gamma-\gamma_{e}\right)+\frac{2(1-\delta)}{E \sqrt{\beta R}} s \\
& =\frac{2 k(1-\delta)}{E \sqrt{\beta R}}\left\{\frac{2\left(\gamma-\gamma_{e}\right)}{\epsilon E}-\left[1+\frac{2(1-\delta)}{\epsilon E \sqrt{\beta R}}\right] s\right\}(62)
\end{aligned}
$$

Finally, we combine Eqs. 60 and 62 into Eq. 57 to get the integrated form for $I(y)$ :

$$
\begin{aligned}
I= & -\frac{\delta}{\beta R}(\ln y)^{2}-\frac{2(1-\delta)}{E \sqrt{\beta R}}[\ln y+k \\
& \left.+\frac{2 k(1-\delta)}{\epsilon E \sqrt{\beta R}}\right] s+\frac{2\left(\gamma-\gamma_{e}\right)}{E}\left[\frac{2 k(1-\delta)}{\epsilon E \sqrt{\beta R}}\right. \\
& \left.+\ln y-\frac{2 k \beta R\left(1-\cos \gamma_{e}\right)}{2 k(1-\delta)+\epsilon E \sqrt{\beta R}}\right]
\end{aligned}
$$

The second-order solution thus consists of Eqs. 38 and 45, with evaluations from Eqs. 48, 53-55, and 63.

\section{Numerical Example}

Now let us consider a Hohmann transfer to Mars. The atmospheric entry velocity is $\delta=0.3911$. Since a higher $\delta$ corresponds to a lower entry velocity, then in general $\delta \leq 0.3911$. A sample flythrough trajectory is shown in Figs. 2 and 3. In this example we use $\epsilon=0.00135$ and $\gamma_{e}=-8^{\circ}$. The atmospheric parameter for Mars is $\beta R=350$.

Figure 2 illustrates the altitude profile as a function of flight path angle. Note that the profile for the first-order solution is symmetric about $\gamma=0$ : the exit flight path angle is the same as the entry flight path angle. In reality, drag forces will lower the exit flight path angle. The integral $I(y)$ produces this effect in the second-order solution, but overestimates the drag losses.

In Fig. 3, $V / V_{c}=\sqrt{2}$ corresponds to the parabolic (escape) condition. The trajectory starts off hyperbolic on the right, and drag forces slow the spacecraft down to an elliptic (captured) orbit. The first-order solution drastically underestimates the exit speed, and is obviously not a good approximation. The second-order solution yields much better results, but still underestimates the exit speed. 


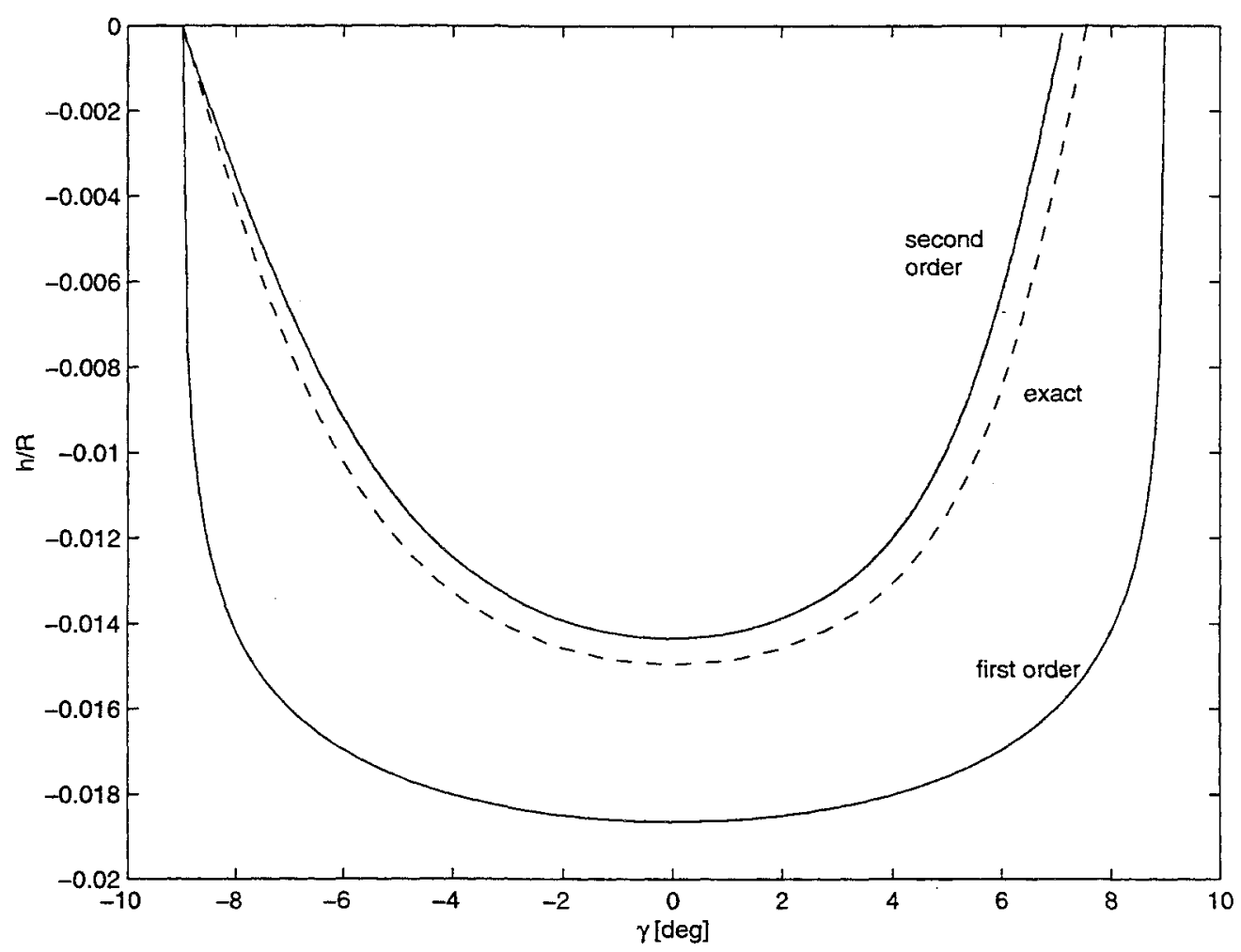

Fig. 2 Plot of trajectory using numerical integration, the first-order solution, and the second-order solution.

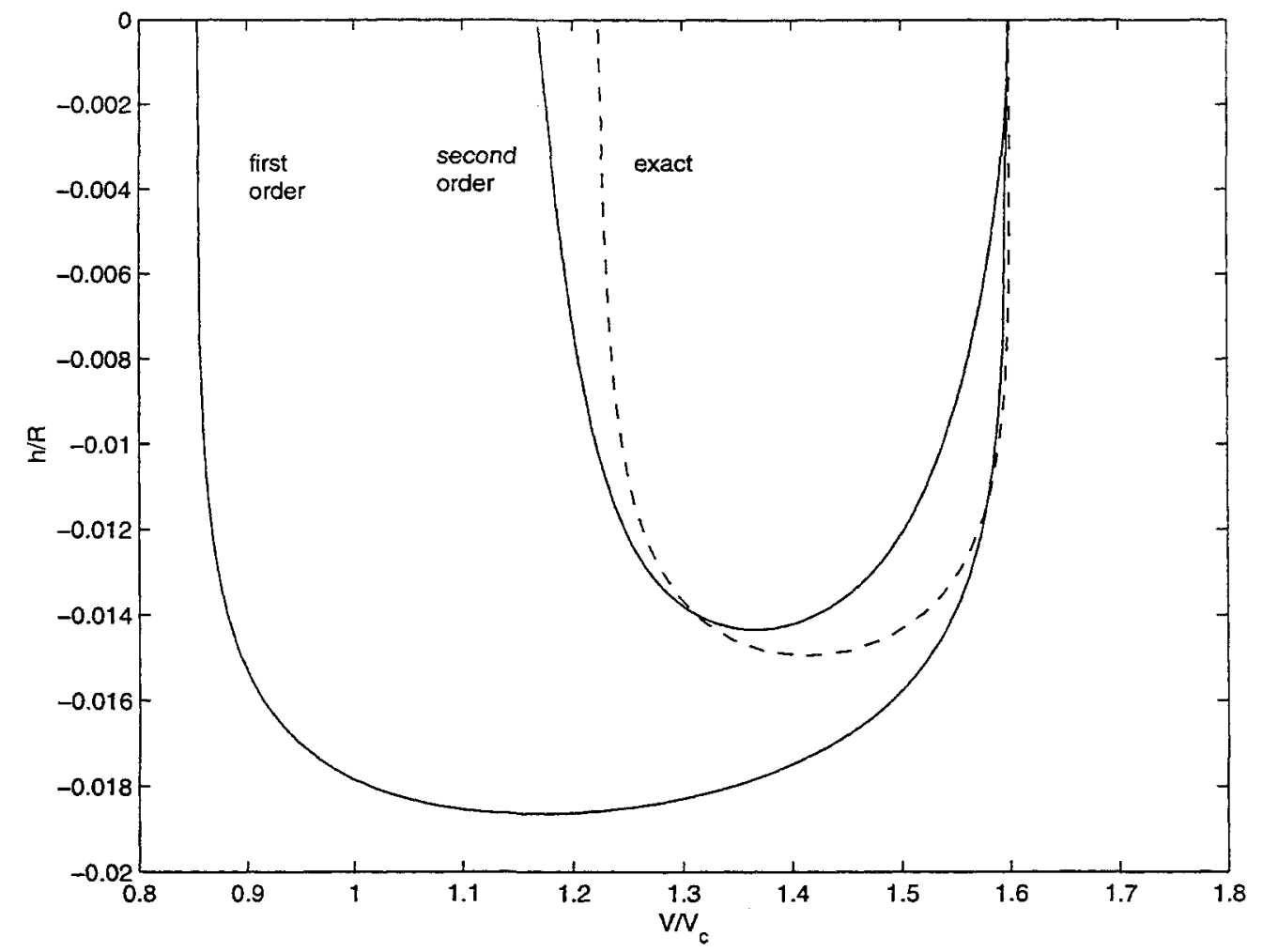

Fig. 3 Plot of trajectory using numerical integration, the first-order solution, and the second-order solution. Since $V / V_{c}$ decreases during the flythrough, the entry point is located at the upper-right, while the exit point is located at the 


\section{Control Law}

The objective of the flythrough is to use aerodynamic forces to minimize the $\Delta V$ required for an insertion into a circular orbit around Mars. If the aerobraking is done in a single atmospheric flyby, then at exit of the Martian atmosphere the final speed $V_{f}$ and final flight path angle $\gamma_{f}$ must be such that apoapsis of the resulting elliptic orbit is at the intended position. At apoapsis, a $\Delta V$ is applied to circularize the orbit. By using the conservation of energy and angular momentum, we see that:

$$
\begin{aligned}
\frac{V_{f}^{2}}{2}-\frac{\mu}{R} & =\frac{V_{a}^{2}}{2}-\frac{\mu}{r_{a}} \\
R V_{f} \cos \gamma_{f} & =r_{a} V_{a}
\end{aligned}
$$

We solve Eq. 65 for $V_{a}$ and substitute into Eq. 64 . Next, we make the substitutions

$$
\begin{aligned}
u_{f} & =\frac{V_{f}^{2}}{\mu / R}=\frac{1}{\delta} e^{-x_{f}} \\
\alpha & =\frac{r_{a}}{R}
\end{aligned}
$$

Thus, at atmospheric exit we have:

$$
u_{f}^{2}\left(\alpha^{2}-\cos ^{2} \gamma_{f}\right)=2 \alpha(\alpha-1)
$$

For a flythrough trajectory, with hyperbolic entry speed and small entry angle, the exit angle is positive, and slightly less in magnitude than the entry angle. Hence, in Eq. 66 we can use the approximation $\cos \gamma_{f} \approx \cos \gamma_{e}$. In our guidance scheme we modulate the bank control $E$ to achieve the required exit speed. From Eq. 44, we see that a small value of $E$ provides more speed depletion.

Following the suggestion by Lyons ${ }^{7}$ for drag modulation, we propose the following guidance law for bank modulation. With a constant lift-to-drag ratio, a given entry speed and entry angle, we select a high bank angle, $\sigma_{1}$, with corresponding value $E_{1}=\left(C_{L} / C_{D}\right) \cos \sigma_{1}$, such that the exit speed is slightly less than the required speed. This trajectory will undershoot the desired apoapsis. During the ascent, a pure lifting trajectory (i.e., $\sigma=0$ ), leads the spacecraft into an overshoot trajectory. Thus, the bank angle can be controlled between these two values $\left(0\right.$ and $\left.\sigma_{1}\right)$ to allow for atmospheric exit at the desired speed. In practice, the spacecraft can fly with a positive bank angle during the atmospheric descent, and switch to the opposite bank angle for the ascent. This will prevent the inclination from changing too much.

\section{First-order Control Law}

To formulate a bank control law, the use of the simplified equations during the ascent may be adequate. Combining Eqs. 22 and 23, we obtain:

$$
\frac{d y}{d x}=-\frac{\sqrt{\beta R}}{\epsilon} \sin \gamma
$$

If we assume a constant mean value for $\sin \gamma \approx$ $\sin \gamma_{m}$ for the integration, we obtain

$$
y-1=-\frac{\sqrt{\beta R}}{\epsilon} \sin \gamma_{m}\left(x-x_{f}\right)
$$

The average value for $\sin \gamma$ can be updated over time, and given as:

$$
\sin \gamma_{m}=(1 / 2)\left(\sin \gamma+\sin \gamma_{f}\right)
$$

Hence, we have:

$$
y-1=-\frac{\sqrt{\beta R}}{2 \epsilon}\left(\sin \gamma+\sin \gamma_{f}\right)\left(x-x_{f}\right)
$$

On the other hand, we recall Eq. 26, with the approximation $\cos \gamma_{e} \approx \cos \gamma_{f}$ to yield:

$$
\frac{\epsilon E}{2}(y-1)=\sqrt{\beta R}\left(\cos \gamma-\cos \gamma_{f}\right)
$$

By eliminating $(y-1)$, we have the required control law:

$$
E=\frac{4 \tan \left[\left(\gamma_{f}-\gamma\right) / 2\right]}{x_{f}-x}
$$

If we assume small angles on Eq. 72, we can make the approximation:

$$
\tan \left(\gamma_{f}-\gamma\right) \approx \gamma_{f}-\gamma
$$

This is nothing more than Eq. 28 integrated from the current state to the exit state. From Figs. 2 and 3 , we see that the first order solution is fairly inaccurate. However, the guidance scheme does not have to rely on exact relationships. As long as the control law is correcting in the right direction, the first-order guidance scheme given by Eq. 72 may work. Also of particular importance is the law's reliance on only two state variables: the flight path angle, and velocity, both of which are easily measurable.

Unfortunately, numerical tests reveal that this simple control law suffers from a few problems. The first problem is that in the ideal case, $E$ is always positive. Targeting errors may make it possible for the spacecraft to skip off the atmosphere before suffcient velocity is lost to capture the spacecraft, where a negative lift would prevent this condition. The second problem is that $x_{f}$ and $\gamma_{f}$ are only known 
for the nominal case. With targeting errors, it is possible that $x$ will at some point exceed $x_{f}$ during the flythrough. This creates a singularity in the denominator. This problem is alleviated by bounding the control to $|E| \leq E_{\max }$. However, this solution causes a side effect - as the singularity is passed, the control will rapidly switch from one extreme to the other. The negative lift induced by this control may help prevent the problem described earlier; otherwise it is inefficient, as the control could have chosen an intermediate value to produce the same effect.

We compute some sample trajectories both using the control, and not using it, for some prescribed nominal conditions, and for several perturbations. Navigational uncertainties may cause up to a $\pm 1^{\circ}$ $(3 \sigma)$ error in the entry flight path angle. ${ }^{8}$ Atmospheric variations may cause up to a $60 \%$ error $(3 \sigma)$ in $\epsilon$, based on parameters given by Tragesser ${ }^{9}$ and Tragesser and Longuski. ${ }^{10}$ The results of the simulations are summarized in Table 1.

\section{Second-order Control Law}

We can deduce a second-order control law by combining Eqs. 48, 53, 54, and 55 into the first three terms on the right-hand side of Eq. 45:

$$
E=\frac{2\left(\gamma_{f}-\gamma\right)-\frac{2(1-\delta)}{\sqrt{\beta R}}\left(s_{f}-s\right)}{x_{f}-x-\frac{2 \delta}{\beta R} \ln y}
$$

Equation 74 is equal to Eq. 72 with the small angle approximation and a correction term applied to both the numerator and denominator. (To keep the calculations simple we use the constant nominal value of $E$ where it appears in Eqs. 53 and 54.) Equation 74 may still have a singularity in the denominator; thus, the bounded constraint still applies. However, since this control is more accurate, the singularity occurs less frequently. Also, we note that this second-order controller requires one more state feedback (the density altitude). A summary of results for the second-order guidance scheme are also included in Table 1.

For the nominal case, the two controllers both erroneously adjust the bank angle, rather than leaving it at the already optimal value. This occurs because the controllers are based on approximations to the EOMs. As expected, the second-order controller which more closely approximates the exact EOMs has a smaller error for the nominal case.

In the cases presented in Table 1, the first-order controller is only occasionally better than the uncontrolled and 2nd-order cases. However, additional testing shows that the 1st-order controller is more
Table 1 Performance of control laws

\begin{tabular}{|c|c|c|c|c|c|}
\hline \multirow[b]{2}{*}{$\begin{array}{l}\text { Entry } \\
\text { Error }\end{array}$} & \multirow[b]{2}{*}{ Control } & \multicolumn{4}{|c|}{ Exit Conditions } \\
\hline & & $\begin{array}{l}\gamma_{f} \\
\text { deg }\end{array}$ & e & $\alpha$ & $\begin{array}{l}\Delta V \\
\mathrm{~m} / \mathrm{s}\end{array}$ \\
\hline \multirow{3}{*}{$\begin{array}{l}\text { nominal }^{a} \\
\text { conditions }\end{array}$} & uncontrolled & 7.54 & 0.50 & 3.00 & 0.0 \\
\hline & 1st-order & 8.15 & 0.56 & 3.51 & 80.9 \\
\hline & 2nd-order & 7.86 & 0.52 & 3.17 & 29.8 \\
\hline \multirow{3}{*}{$+60 \% \epsilon$} & uncontrolled & 7.43 & 0.41 & 2.40 & 131.2 \\
\hline & 1st-order & 8.18 & 0.48 & 2.81 & 37.7 \\
\hline & 2nd-order & 7.64 & 0.46 & 2.72 & 55.0 \\
\hline \multirow{3}{*}{$+40 \% \epsilon$} & uncontrolled & 7.45 & 0.44 & 2.55 & 94.4 \\
\hline & 1st-order & 8.21 & 0.50 & 2.98 & 4.3 \\
\hline & 2nd-order & 7.97 & 0.48 & 2.85 & 30.0 \\
\hline \multirow{3}{*}{$+20 \% \epsilon$} & uncontrolled & 7.50 & 0.46 & 2.74 & $\overline{51.8}$ \\
\hline & 1st-order & 8.19 & 0.52 & 3.20 & 34.4 \\
\hline & 2nd-order & 8.07 & 0.50 & 2.99 & 1.8 \\
\hline \multirow{3}{*}{$-20 \% \epsilon$} & uncontrolled & 7.61 & 0.54 & 3.39 & 64.4 \\
\hline & 1st-order & 8.09 & 0.60 & 3.96 & 138.6 \\
\hline & 2nd-order & 7.66 & 0.55 & 3.45 & 73.4 \\
\hline \multirow{3}{*}{$-40 \% \in$} & uncontrolled & 7.69 & 0.60 & 4.06 & 150.2 \\
\hline & 1st-order & 8.04 & 0.65 & 4.71 & 213.1 \\
\hline & 2nd-order & 7.51 & 0.59 & 3.93 & 136.1 \\
\hline \multirow{3}{*}{$-60 \% \epsilon$} & uncontrolled & 7.84 & 0.69 & 5.55 & 274.5 \\
\hline & 1st-order & 7.98 & 0.73 & 6.35 & 319.6 \\
\hline & 2nd-order & 7.44 & 0.67 & 5.01 & 237.5 \\
\hline \multirow{3}{*}{$+1^{\circ} \gamma_{e}$} & uncontrolled & 7.22 & 0.83 & 10.81 & 456.4 \\
\hline & 1st-order & 7.22 & 0.85 & 12.14 & 478.7 \\
\hline & 2nd-order & 6.64 & 0.79 & 8.40 & 399.4 \\
\hline \multirow{3}{*}{$+0.5^{\circ} \gamma_{e}$} & uncontrolled & 7.40 & 0.66 & 4.91 & 229.2 \\
\hline & 1st-order & 7.64 & 0.70 & 5.66 & 281.9 \\
\hline & 2nd-order & 7.04 & 0.64 & 4.51 & 195.6 \\
\hline \multirow{3}{*}{$-0.5^{\circ} \gamma_{e}$} & uncontrolled & 7.62 & 0.35 & 2.09 & 223.4 \\
\hline & 1st-order & 7.14 & 0.41 & 2.39 & 134.4 \\
\hline & 2nd-order & 6.85 & 0.40 & 2.36 & 141.2 \\
\hline \multirow{3}{*}{$-1^{\circ} \gamma_{e}$} & uncontrolled & 7.67 & 0.22 & 1.58 & 436.8 \\
\hline & 1st-order & \multicolumn{4}{|c|}{. crash . } \\
\hline & 2nd-order & 4.92 & 0.26 & 1.72 & 357.1 \\
\hline
\end{tabular}

robust than the uncontrolled case. For cases where the nominal eccentricity is close to 1 , and there is a small margin for error, the first-order controller tends to capture the spacecraft where it would otherwise escape. With the exception of the thicker-thanexpected atmospheres, the second-order controller performs the best.

Figure 4 shows the difference between the actual velocity and nominal velocity during a flythrough for the nominal case (see Table 1). By Eq. 66, apoapsis is almost entirely determined by final velocity. Thus, this plot gives us a means to see how well the control is behaving for the different cases. For the two nominal cases, both controls begin to introduce errors in the flight profile, but the second-order control is better at returning to the nominal trajectory. For the thin atmosphere, all cases are forced to descend using the fixed $E$, which is too high for this case. At periapsis, the second-order controller applies a proper correction, but is unable to arrest 


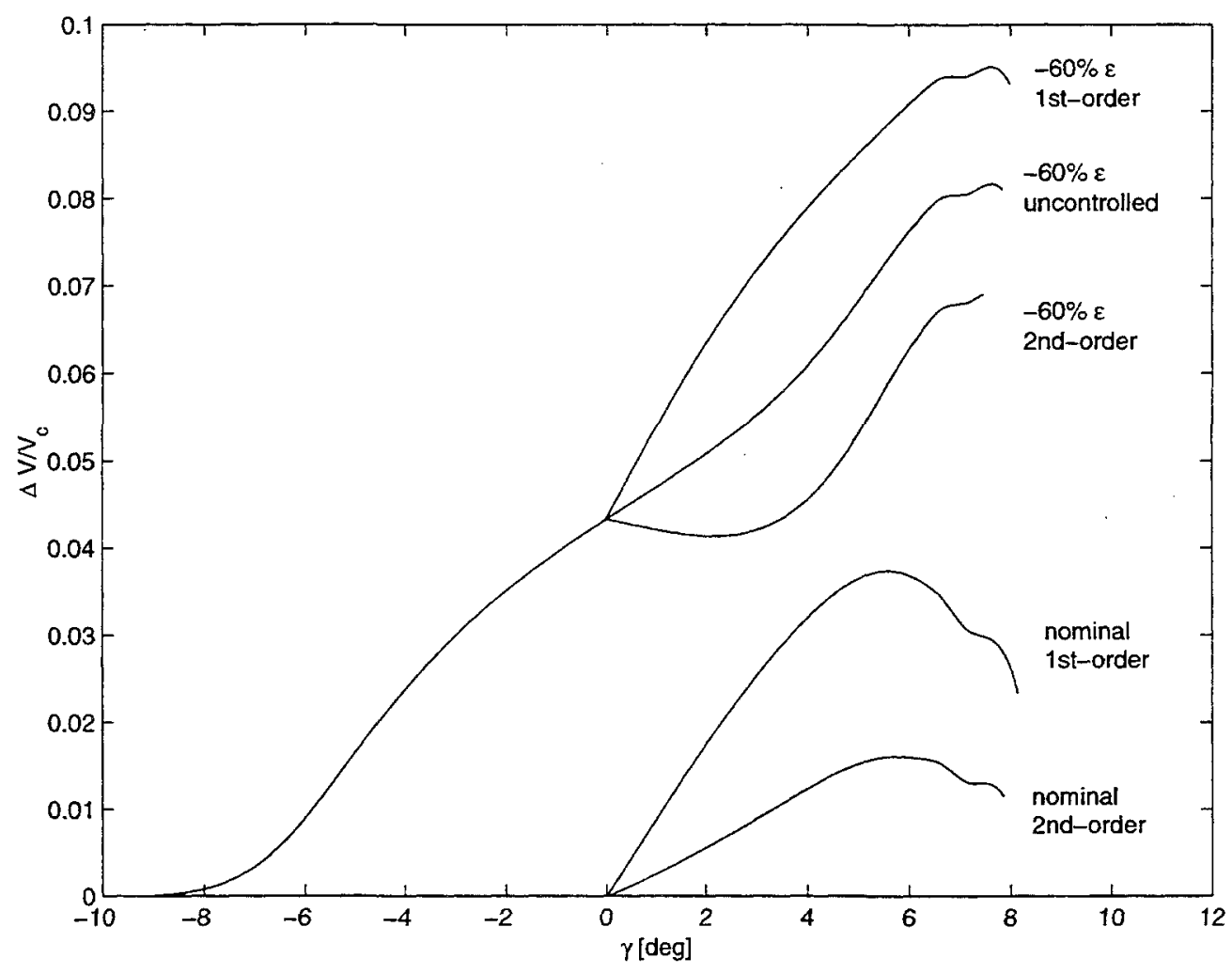

Fig. 4 Difference between actual velocity and nominal velocity for several cases.

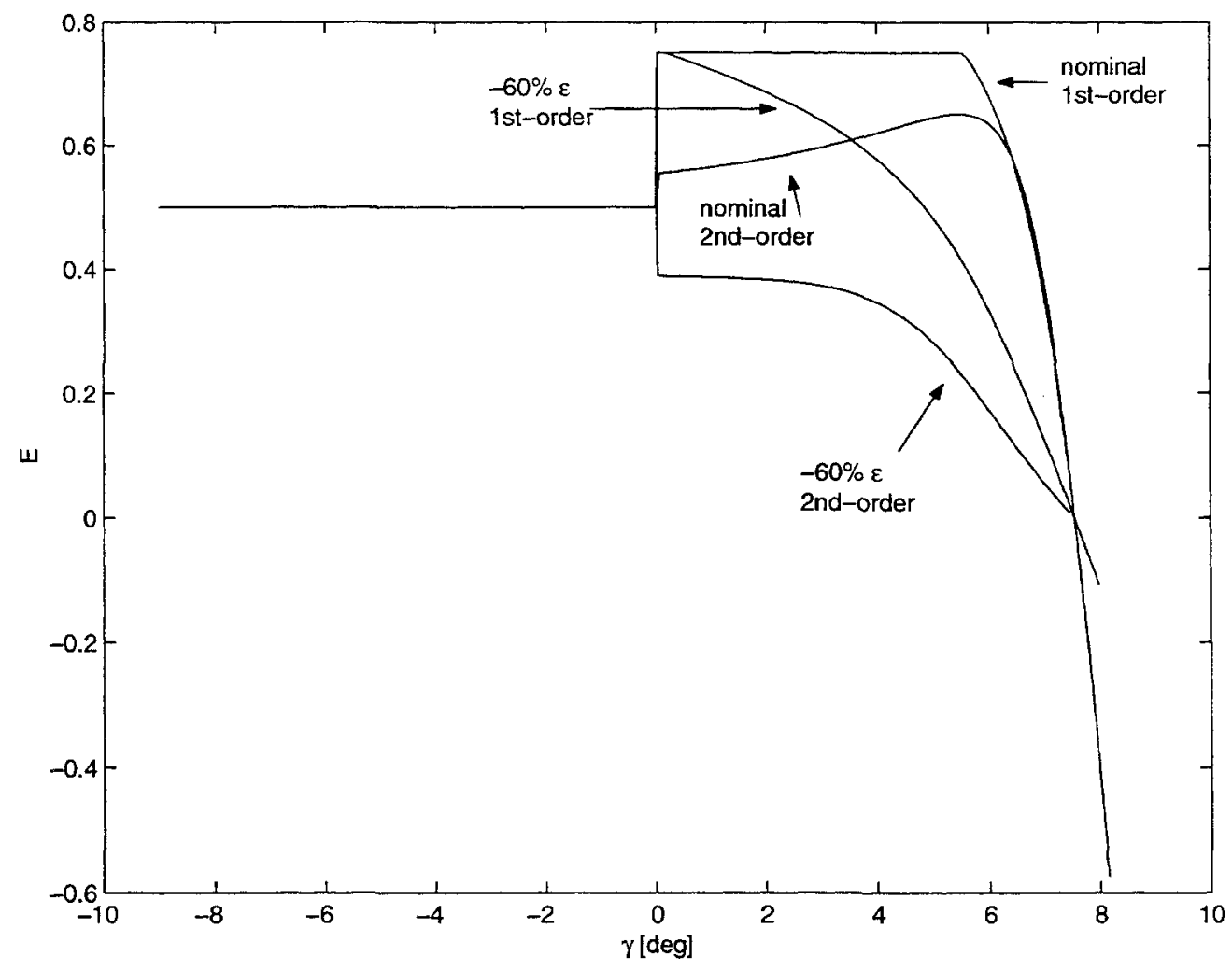

Fig. 5 Controls for several cases. $(L / D)_{\max }=0.75$. 
its ascent in time to return to the nominal condition. Unfortunately, the first-order controller applies a correction in the wrong direction, and only begins a proper correction just before the spacecraft exits the atmosphere.

Figure 5 shows the control for these same cases. (The uncontrolled trajectories simply have a constant $E=0.5$ ). Again, this plot shows that the second-order controller is quicker than the first-order controller to adjust its $E$ to a smaller value. For the thin atmosphere, the first-order controller begins in the wrong direction, and only begins to compensate at the very end.

Several investigators (Braun and Powell, ${ }^{11,12}$ Thorp and Pierson, ${ }^{4}$ Gurley, ${ }^{13}$ and Evans and Dukeman ${ }^{14}$ ) have explored sophisticated guidance schemes in applications for missions that have been flown or are currently being planned. We do not expect our simple guidance laws (in their present form) to compete with these elaborate algorithms, but they may point the way to a new approach as we investigate them further.

\section{Conclusions}

The analytic theory for aerocapture of a lifting body provides the basis for a guidance law to control the atmospheric exit conditions. We develop two laws for bank modulation, which are relatively simple to perform. Numerical tests of the guidance schemes demonstrate that errors in entry conditions (larger than typical delivery errors) and large uncertainties in atmospheric density are accommodated. These techniques may stimulate the development of more robust aerocapture techniques for future interplanetary missions.

\section{References}

${ }^{1}$ Vinh, N., Johannesen, J., Longuski, J., and Hanson, J., "Second-Order Analytical Solution for Aerocapture and Ballistic Fly-Through Trajectories," Journal of the Astronautical Sciences, Vol. 32, No. 4, Oct.-Dec. 1984, pp. 429-445.
${ }^{2}$ Vinh, N., Johannesen, J., Mease, K., and Hanson, J., "Explicit Guidance of Drag Modulated Aeroassisted Transfer between Elliptical Orbits," AIAA Journal of Guidance, Control, and Dynamics, Vol. 3, No. 3, May-June 1986, pp. 274280 .

${ }^{3}$ Lee, W., D'Amario, L., Roncoli, R., and Smith, J., "Mission Design Overview for 2003/2005 Mars Sample Return Mission," AAS Paper 99-305, AAS/AIAA Astrodynamics Specialist Conference, Girdwood, Alaska, Aug. 1999.

${ }^{4}$ Thorp, N. and Pierson, B., "Robust Roll Modulation Guidance for Aeroassisted Mars Mission," AIAA Journal of Guidance Control and Dynamics, Vol. 18, No. 2, March-April 1995, pp. 298-305.

${ }^{5}$ Vinh, N., Busemann, A., and Culp, R., Hypersonic and Planetary Entry Flight Mechanics, The University of Michigan Press, Ann Arbor, 1980.

${ }^{6}$ Eggers, Jr, A., Allen, H., and Neice, S., "A Comparative Analysis of the Performance of Long-Range Hypervelocity Vehicles," NACA TN 4046, 1957.

${ }^{7}$ Lyons, D., "Aerobraking at Venus and Mars, a Comparison of the Magellan and Mars Global Surveyor Aerobraking Cases," AAS Paper 99-358, AAS/AIAA Astrodynamics Conference, Girdwood, Alaska, Aug. 1999.

${ }^{8}$ Spencer, D. and Braun, R., "Mars Pathfinder Atmospheric Entry: Trajectory Design and Dispersion Analysis," Journal of Spacecraft and Rockets, Vol. 33, No. 5, SeptOct. 1996, pp. 670-676.

${ }^{9}$ Tragesser, S., "Analysis and Design of Aerobraking Tethers," Ph.D. Thesis, School of Aeronautics and Astronautics, Purdue University, West Lafayette, IN, Dec. 1997.

${ }^{10}$ Tragesser, S. and Longuski, J., "Analysis and Design of Aerocapture Tether with Accounting for Stochastic Errors," Journal of Spacecraft and Rockets, Vol. 35, No. 5, Sept.-Oct. 1998, pp. 683-689.

${ }^{11}$ Braun, R. and Powell, R., "Predictor-Corrector Guidance Algorithm for Use in High-Energy Aerobraking Systems Studies," Journal of Guidance, Control, and Dynamics, Vol. 15, No. 3, May-June 1992, pp. 672-678.

${ }^{12}$ Powell, R. and Braun, R., "Six-Degree-of-Freedom Guidance and Control Analysis of Mars Aerocapture," Journal of Guidance, Control, and Dynamics, Vol. 16, No. 6, Nov.-Dec. 1993, pp. 1038-1044.

${ }^{13}$ Gurley, J., "Guidance for an Aerocapture Maneuver," Journal of Guidance, Control, and Dynamics, Vol. 16, No. 3, May-June 1993, pp. 505-510.

${ }^{14}$ Evans, S. and Dukeman, G., "Examination of a Practical Aerobraking Guidance Algorithm," Joumal of Guidance, Control, and Dynamics, Vol. 18, No. 3, May-June 1995, pp. $471-477$. 